\title{
Detection of Extensive Metastases from Anaplastic Thyroid Cancer by F- 18 FDG-PET/CT
}

\author{
M. Vorster and M.M. Sathekge*
}

Department of Nuclear Medicine, University of Pretoria and Steve Biko Academic Hospital, Pretoria, South Africa

\begin{abstract}
Background: Survival with anaplastic thyroid cancer is dependent on how rapid multimodality treatment (chemotherapy, radiotherapy and surgery) and thus accurate assessment of the extent of the disease critical to predict prognosis and therapy planning.

Methods \& Results: We present a case of anaplastic cancer in a 54-year-old woman who presented with a supra-sternal lump which has decreased in size in response to chemo- and radiotherapy. CT and FDG-PET/CT scan were performed after therapy. CT demonstrated supra-sternal and lung metastases. FDG-PET/CT demonstrated the primary lesion and its distant widespread metastases, beyond the lung and supra-sternal mass.

Conclusion: This case emphasizes the need for FDG PET/CT if there is equivocal clinical, laboratory, and radiological evidence of recurrence and metastases, especially if the suspected finding seems can be very unusual for metastatic lesions.
\end{abstract}

Keywords: Thyroid, anaplastic cancer, metastases, FDG PET/CT.

\section{INTRODUCTION}

Survival with anaplastic thyroid cancer is dependent on early initiation of multimodality treatment (chemotherapy, radiotherapy and surgery) and thus accurate assessment of the extent of the disease is critical to therapy planning.

We present a case of anaplastic cancer in a female patient who was referred for PET/CT to assess response to chemotherapy.

This case emphasizes the need for FDG PET/CT if there is equivocal clinical, laboratory, and radiological evidence of recurrence and metastases, especially if the suspected findings seem unusual for metastatic lesions.

\section{PATIENT HISTORY \& CLINICAL PRESENTATION}

54 yr old female with confirmed anaplastic thyroid cancer who underwent a partial thyroidectomy upon diagnosis which was followed by radiotherapy and chemotherapy. The last radiotherapy was received a year prior to the scan. Medication taken at the time of the PET/CT included oral chemotherapy and Eltroxin.

A recent CT performed prior to the PET/CT demonstrated a supra-sternal mass as well as lung metastases.

Patient symptoms consisted of fatigue, weight loss and dysphagia. The supra-sternal mass had decreased in size following chemo- and radiotherapy. Clinically, this finding implied a good response to therapy.

*Address correspondence to this author at the Department of Nuclear Medicine, University of Pretoria and Steve Biko Academic Hospital, Pretoria, South Africa; Tel + 2712354 1794; Fax: + 2712354 1219; E-mails: mike.sathekge@up.ac.za or sathekgemm@yahoo.com

\section{F-18-FDG PET/CT IMAGES}

\section{PET Findings}

Fusion images (1A) demonstrate the presence of local disease and multiple widespread metastatic lesions. PET (1B) show a primary thyroid mass with an irregular contour (SUV max: 6.24)

In addition, the FDG PET/CT scan showed a metastatic suprasternal soft tissue mass (1C), multiple lung lesions, mediastinal lymph nodes and diffuse intense FDG uptake throughout the lower oesophagus (1D) and most of the liver with sub-hepatic lymph node involvement (1E).

\section{PET/CT Conclusion}

Metabolically active thyroid CA with widespread metastatic disease involving soft tissue, the lungs, mediastinal- and abdominal lymph nodes, the esophagus, stomach and liver.

The GIT involvement was unexpected and thus changed the envisaged hope to therapy response and future management strategy.

\section{LITERATURE REVIEW \& DISCUSSION}

Anaplastic thyroid cancers (ATC) are a histologically heterogeneous group of extremely aggressive undifferentiated tumours arising from the follicular epithelium which accounts for $2-5 \%$ of all thyroid cancers. ATC cells do not retain any of the biological features of the original follicular cells such as uptake of Iodine and synthesis of thyroglobulin, but still remains metabolically active causing intense uptake on FDG. 


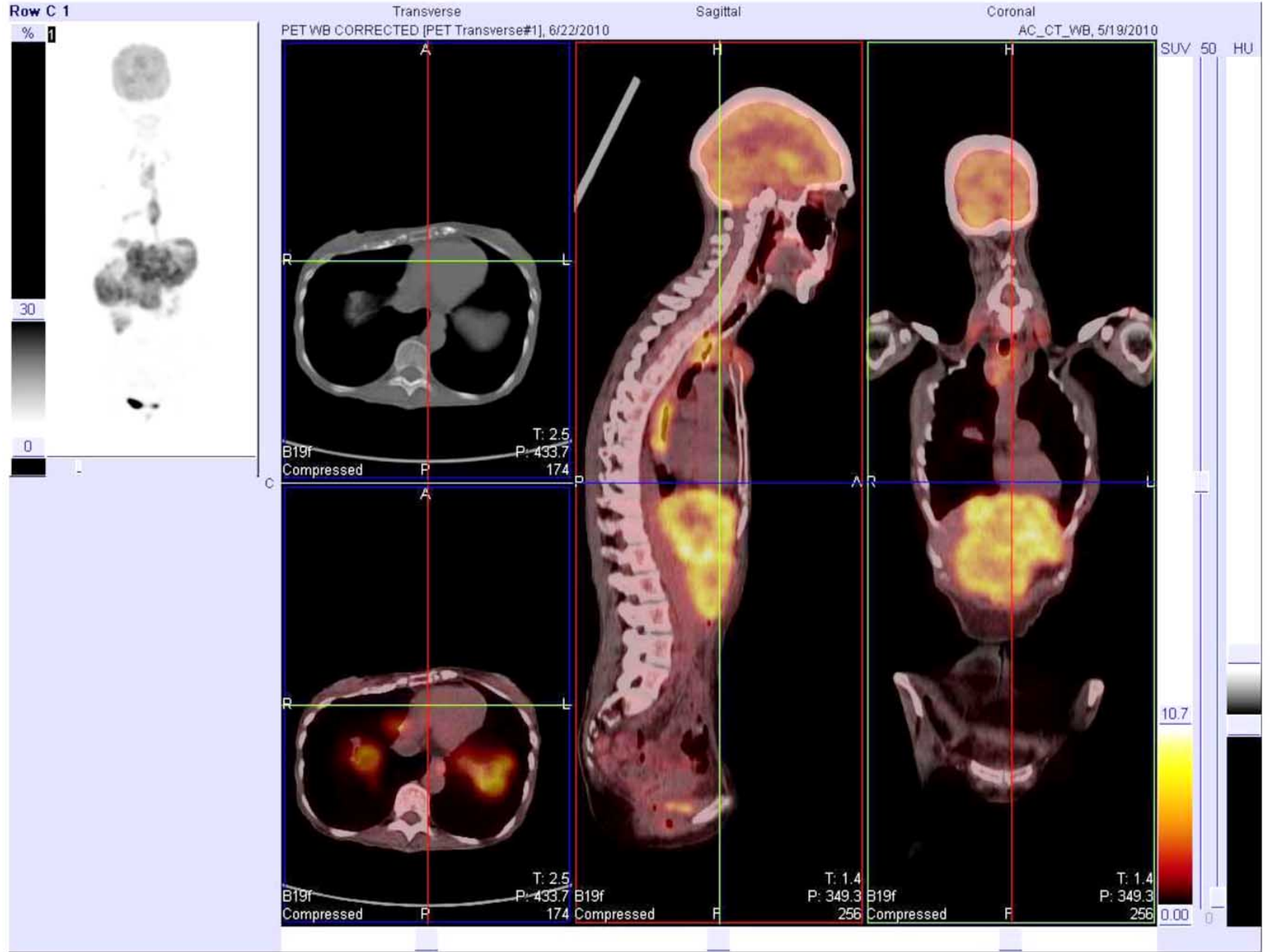

Fig. (1A). Local thyroid uptake with widespread metastatic disease.
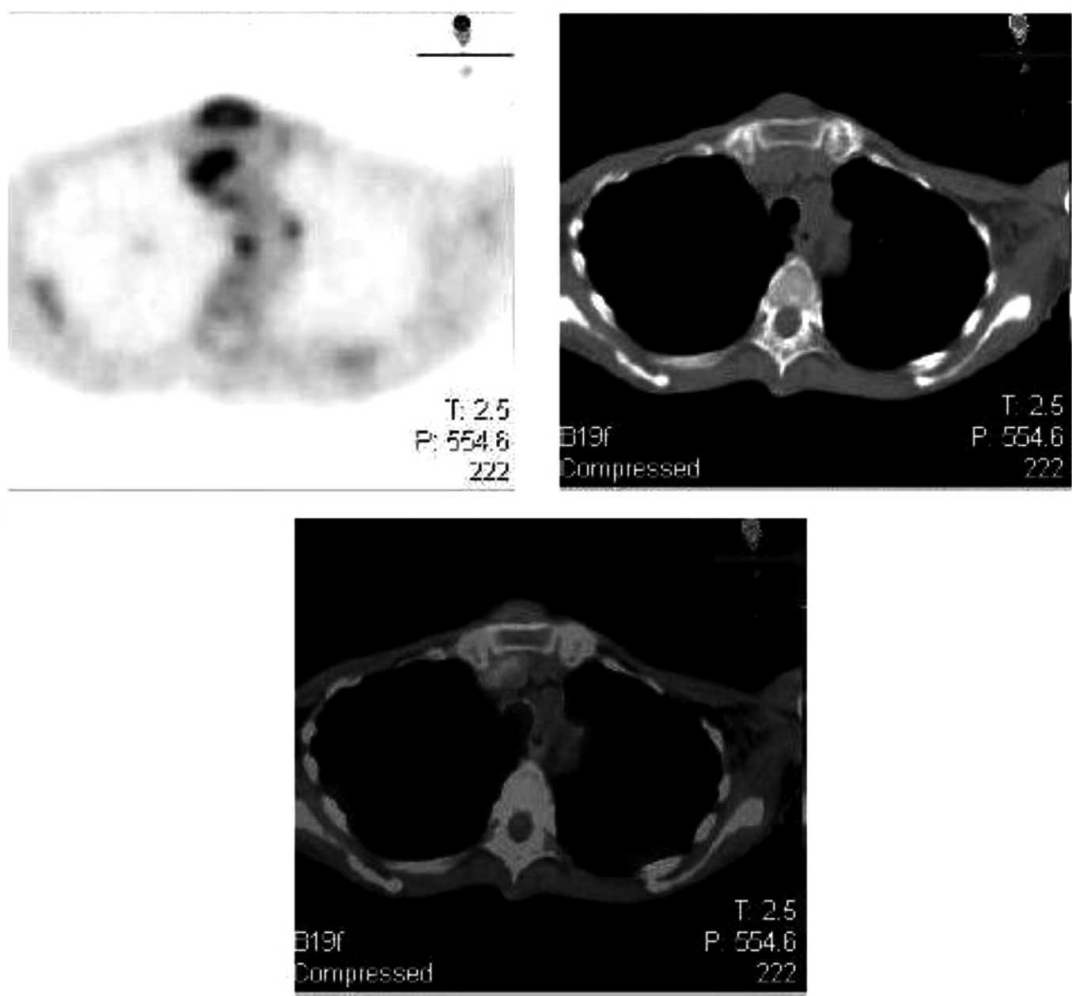

Fig. (1B). Thyroid uptake and mediastinal lymph node involvement. 

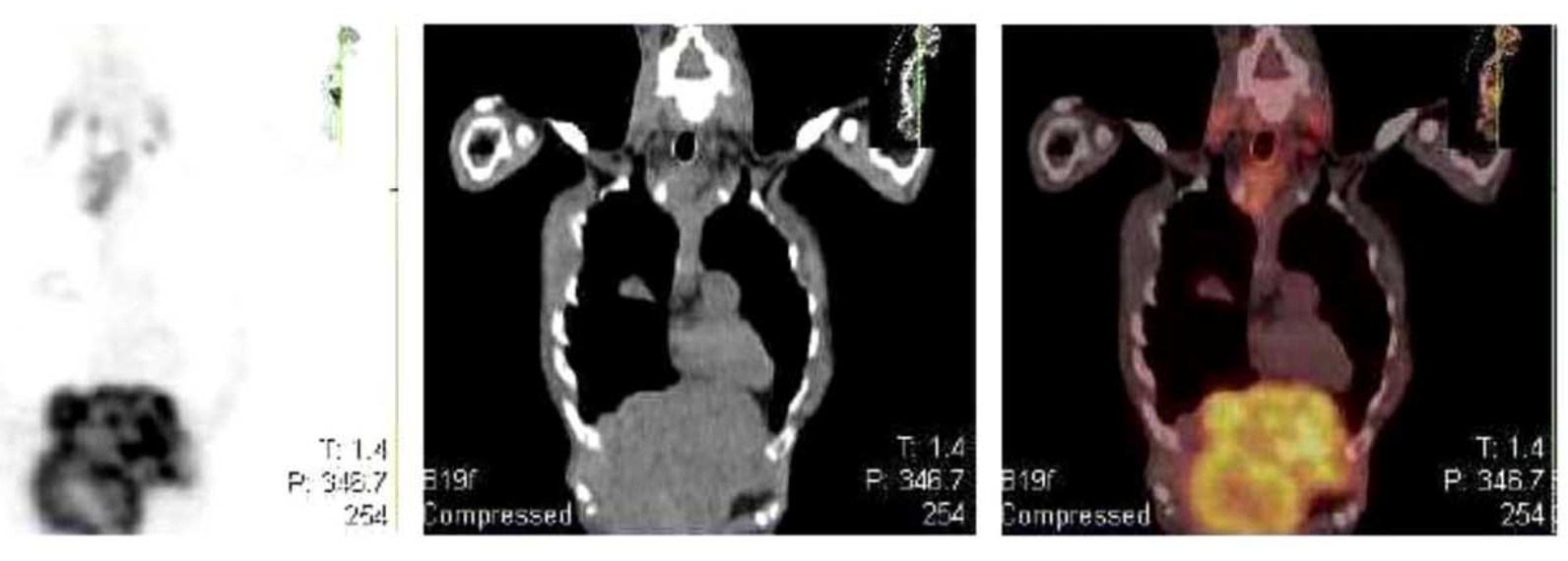

Fig. (1C). Suprasternal mass.
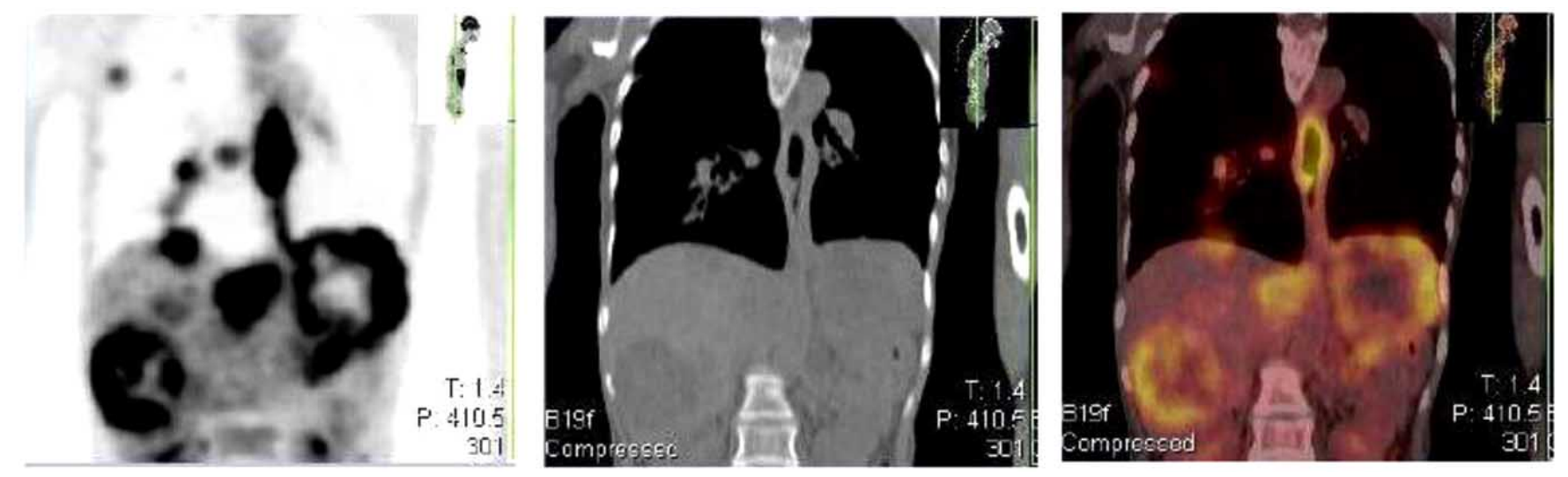

Fig. (1D). Lung metastases, mediastinal lymph nodes, esophagus and stomach involvement.
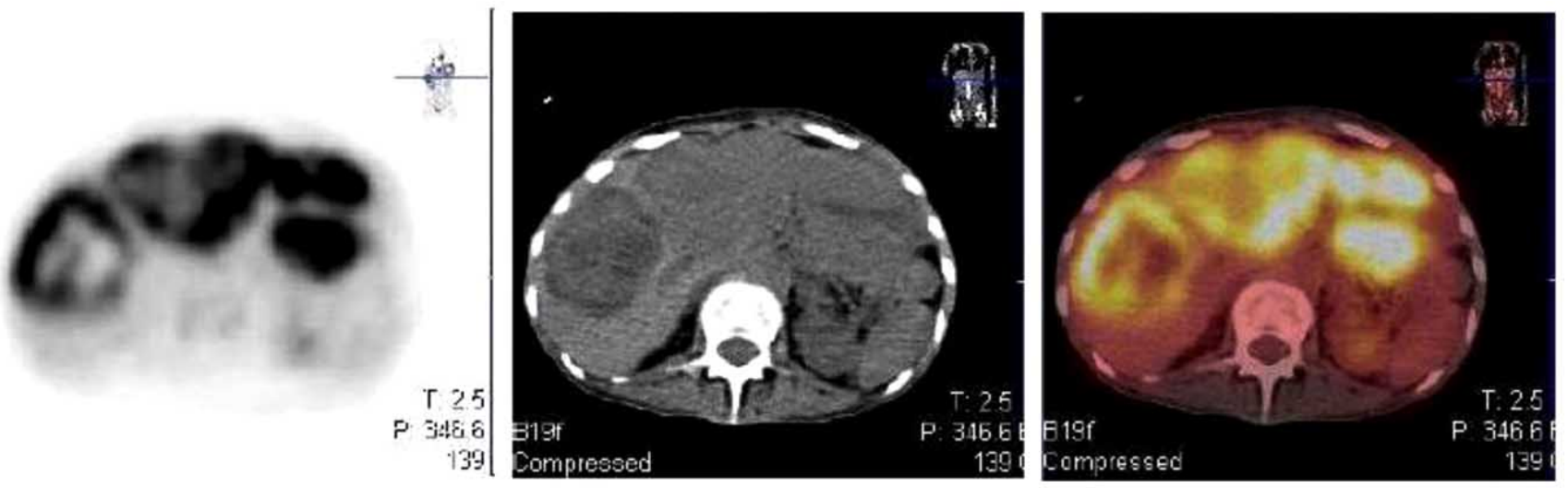

Fig. (1E). Extensive liver metastases and sub-hepatic lymph node mass.

Despite its rare occurrence, it represents over half of thyroid cancer related deaths and prognosis remains poor even with combination therapy. This emphasizes the need for new treatment modalities and early recognition of disease in order to allow prompt therapy initiation. Imaging with FDG-PET/CT could potentially play an important role in evaluating the efficacy of various new treatments.

\section{EPIDEMIOLOGY}

The mean age at diagnosis is 65 yrs (with fewer than $10 \%$ found in patients under the age of $50 \mathrm{yrs})$ and the majority (60-70\%) occurs in women [1-3].
Regional or distant spread is apparent at the time of diagnosis in $90 \%$ of patients and almost all present with a thyroid mass [4-7].

See Figs. (2) and (3) for common disease presentation and clinical manifestations. Our patient presented with some of the less common symptoms and with rare metastatic sites.

\section{CLINICAL MANIFESTATIONS}

Most ATC patients complain of local compressive symptoms, such as dysphagia, dysphonia, stridor and dyspnea in addition to neck pain and tenderness. In over $70 \%$ of the patients the tumor infiltrates surrounding tissues, 


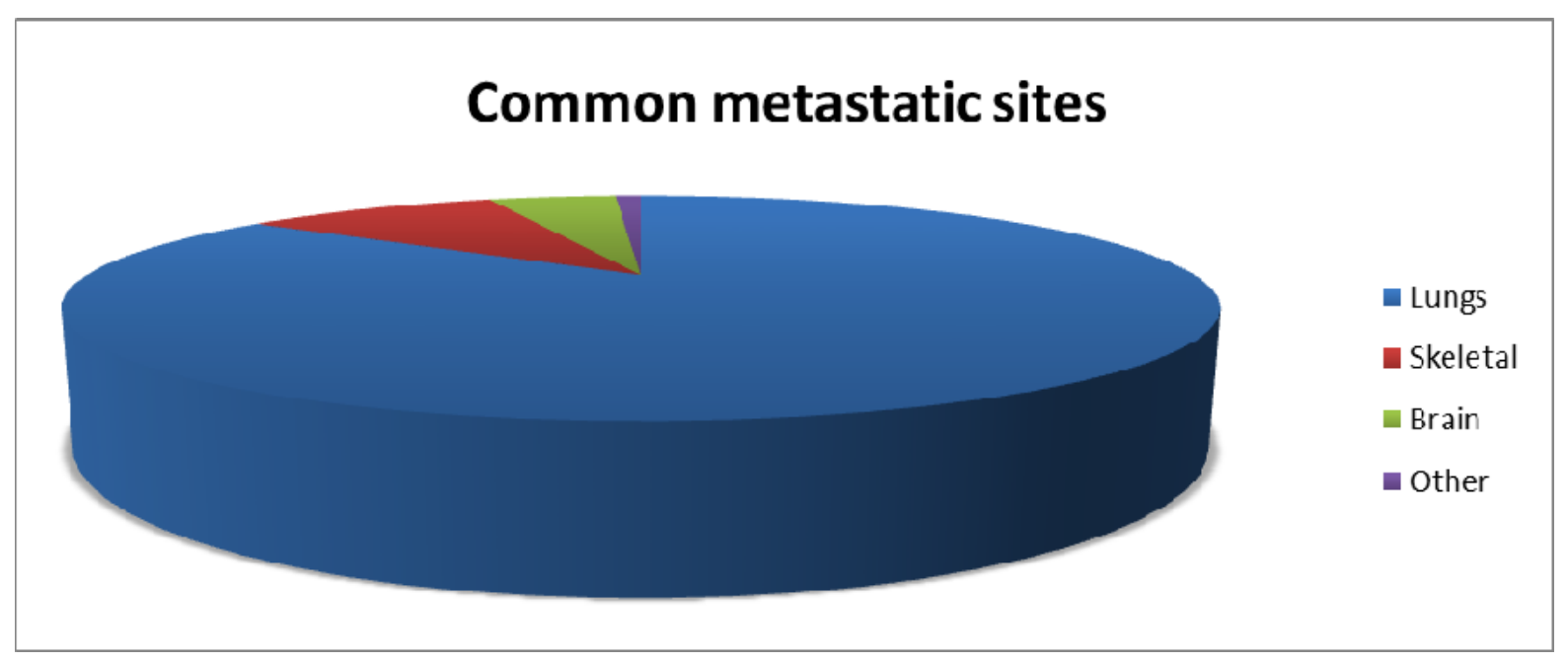

Fig (2). Bar chart demonstrating the lungs as the most common metastatic site.

such as fat, trachea, muscle, esophagus, and larynx. The clinical course of a rapidly enlarging mass that is firm and fixed to surrounding structures in an elderly patient is quite suggestive for ATC.

\begin{tabular}{|l|l|}
\hline Symptom & $\mathbf{\%}$ \\
\hline Rapidly enlarging neck mass & 85 \\
\hline Dyspnoea & 35 \\
\hline Dysphagia & 30 \\
\hline Hoarseness & 25 \\
\hline Cough & 25 \\
\hline $\begin{array}{l}\text { Chest pain, bone pain, headache, confusion, } \\
\text { abdominal pain, constitutional symptoms, } \\
\text { thyroiditis, hyperthyroidism }\end{array}$ & common \\
\hline
\end{tabular}

Fig. (3). Clinical manifestation with rapidly enlarging mass as the commonest presentation.

\section{PATHOGENESIS}

Research into the pathogenesis of anaplastic thyroid cancer has implicated mutations in the p-53 tumour suppression protein, 16p, catenin, beta 1, BRAF, AKT1 and PIK3CA [8-13].

Another hypotheses describes the development of anaplastic thyroid CA from more differentiated tumours (20\% have a history of differentiated thyroid cancer) [14-17].

Recognition of the different clinicopathologic features is important to the optimal management of these tumours, especially in light of emerging molecular targeted therapies which can be tailored to individual patients.

\section{DIAGNOSIS \& STAGING}

Diagnosis is usually confirmed by fine needle aspiration cytology or, in doubtful cases, by histology on core biopsy.

Most patients have normal serum thyroid hormone and thyrotropin (TSH) concentrations, except for those few patients with tumor-related thyroiditis and hyperthyroidism from presumed rapid tumor growth and concomitant tissue destruction [18-20] Serum thyroglobulin concentrations may be high, most often due to secretion from a coexisting differentiated cancer rather than the anaplastic cancer.

Anaplastic thyroid cancer cells will rarely stain positive for thyroid transcription factor-1 (TTF1) or thyroglobulin, but will commonly stain positive for PAX8, which is not positive in carcinomas from other sites. Overall, the pathologic diagnosis can be difficult and may depend partly on the clinical scenario.

BRAF and p53 mutations are common in anaplastic thyroid cancer and may be interesting targets for future investigations.

Imaging with CT, MRI and FDG-PET/CT are useful for evaluating the local extent of disease and for identifying distant metastases.

Tracheoscopy and esophagoscopy should be performed every two months, or whenever patients refer the appearance or worsening of local symptoms.

Bone scintigraphy may be included in the follow-up of patients in relatively good health with a longer survival expectation.

According to the latest American Joint Committee on Cancer Staging Manual all ATSs are classified as T4 and Stage IV tumors, regardless of their actual overall tumor burden [21].

\section{THERAPY}

Although the treatment of ATC has not been standardized it clearly requires a multi-modality approach consisting of surgery, chemotherapy and radiotherapy early on.

Surgical treatment of local disease offers the best opportunity for prolonged survival if the tumor is intrathyroidal. When the tumor is extrathyroidal, the surgical approach to ATC is controversial.

Maintaining airway patency is of paramount importance and a tracheostomy should be performed in patients with impending airway obstruction. Interventional bronchoscopy, including Nd-YAG laser and airways stenting are 
alternatives to surgery in inoperable ATC-induced tracheal obstruction [21].

Some favourable results have recently been reported with newly developed chemotherapy agents and hyper-fractioned radiation therapy.

Preliminary data from a recent study done in a number of patients with ATC suggest that the combination of radiation and concomitant docetaxel is highly effective [22].

Other recent developments include PPARg agonists, adenovirus-mediated p53 tumour suppressor gene therapy and BMP-7 [23].

Advances in our understanding of the many critical genes and proteins affected in Anaplastic thyroid CA and the extensive array of targeted therapies being developed for cancer patients, there are new opportunities to design clinical trials based upon tumor molecular profiling and preclinical studies of potentially synergistic combinatorial novel therapies [24].

\section{ROLE OF IMAGING}

Especially in high-risk patients, aside from the use of ultrasonography for the detection of local recurrence and cervical lymph node metastases, nuclear medicine methods such as radioiodine imaging and FDG-PET are the methods of choice for localizing metastatic thyroid malignancies.

FDG-PET is a valuable tool to detect recurrences and metastases of thyroid cancer, especially in the clinical setting of elevated or rising Thyroglobulin despite a negative 131I WBS.

FDG PET/CT has shown high sensitivity and specificity in staging and re-staging of thyroid cancer and is being increasingly used for detection of local or distal recurrence in poorly differentiated thyroid cancer that is not I-131 avid $[25,26]$.

High to very intense FDG uptake has been demonstrated in primary ATC, residual tumours, recurrent disease, lymph node and extranodal metastases. This has been found to correlate with an overexpression of GLUT-1 on the cell membrane of thyroid cancers [27].

According to a recent studies, PET findings impacted directly on the management of $25-50 \%$ of thyroid cancer patients. PET may therefore improve disease detection and impact significantly on the management of patients with ATC relative to other imaging modalities [27, 28].

Furthermore FDG PET/CT seems promising in the early evaluation of treatment response and follow-up [28].

\section{Prognostic Role}

FDG-PET is able to identify patients at high risk requiring more aggressive treatment using GLUT-1 expression as an in vitro marker [28].

Once distant metastases are discovered in patients with differentiated thyroid carcinoma, FDG-PET can accurately identify high and low risk subsets.

Multivariate analysis performed in a recent study demonstrated that the single strongest predictor of survival was the volume of FDG-avid disease (a volume of less than
$125 \mathrm{ml}$ associated with improved survival) [29]. While a recent study has demonstrated that the volume of FDG uptake $(\geq 300 \mathrm{ml})$ and the intensity of FDG uptake $\left(\mathrm{SUV}_{\max }\right.$ $\geq 18$ ) were significant prognostic factors for survival [28].

A negative PET scan after completed therapy may be indicative of extended survival and persistent FDG uptake during treatment may be indicative of a clinical recurrence and death $[28,29]$.

\section{CONCLUSION}

Imaging with FDG-PET/CT has a well-established role in the management of the patient with Iodine negative metastatic disease with rising Thyroglobulin levels. It has a developing role in the staging and restaging of dedifferentiated thyroid cancer; it may improve disease detection and have an impact on patient management in patients with ATC relative to other imaging modalities.

The use of FDG-PET/CT in patients with anaplastic thyroid cancer may lead to an increase in the understanding of the pathogenesis of this disease as well as evaluating the effectiveness of emerging molecular-based treatment modalities. Furthermore, it can be used to guide treatment by classifying patients into low risk and high risk categories, by evaluating response to therapy and giving an indication of prognosis.

Our case emphasizes the need for FDG PET/CT if there is equivocal clinical, laboratory, and radiological evidence of recurrence and metastases, especially if the suspected finding seems can be very unusual for metastatic lesions.

This case also brings into focus the role of FDG PET/CT in the surveillance of treated patients with anaplastic thyroid cancer and its ability to detect metastatic recurrences at unusual and sites.

\section{REFERENCES}

[1] Mazzaferri EL. Undifferentiated thyroid carcinoma and unusual thyroid malignancies. In: Mazzaferri EL, Samaan NA (eds) Endocrine tumors. Boston: Blackwell. Scientific Publications, 1993; 98: 378-98.

[2] Akslen LA, Haldorsen T, Thoresen SØ, Glattre E. Incidence of thyroid cancer in Norway 1970-1985. APMIS 1990; 98: 549-58.

[3] Kebebew E, Greenspan FS, Clark OH, et al. Anaplastic thyroid carcinoma. Cancer 2005; 103: 1330-5.

[4] Tan RK, Finley RK, Driscoll D, et al. Anaplastic carcinoma of the thyroid: A 24-year experience. Head Neck 1995; 17:41-7.

[5] McIver B, Hay ID, Giuffrida DF, et al. Anaplastic thyroid carcinoma: a 50-year experience at a single institution. Surgery 2001; 130: 102834.

[6] Aldinger KA, Samaan NA, Ibanez ML, Hill CSJ. Anaplastic carcinoma of the thyroid: A review of 84 cases of spindle and giant cell carcinoma of the thyroid. Cancer 1978; 41: 2267-75.

[7] Tennvall J, Lundell G, Wahlberg $\mathrm{P}$, et al. Anaplastic thyroid carcinoma: three protocols combining doxorubicin, hyperfractionated radiotherapy and surgery. Br J Cancer 2002; 86: 1848-53.

[8] Nakamura T, Yana I, Kobayashi T, et al. p53 gene mutations associated with anaplastic transformation of human thyroid carcinomas. Jpn J Cancer Res 1992; 83: 1293-98.

[9] Ito $\mathrm{T}$, Seyama $\mathrm{T}$, Mizuno $\mathrm{T}$, et al. Unique association of $\mathrm{p} 53$ mutations with undifferentiated but not with differentiated carcinomas of the thyroid gland. Cancer Res 1992; 52: 1369-71.

[10] Ito T, Seyama T, Mizuno T, et al. Genetic alterations in thyroid tumor progression: Association with p53 gene mutations. Jpn J Cancer Res 1993; 84: 526-31.

[11] Moretti F, Farsetti A, Soddu S, et al. p53 re-expression inhibits proliferation and restores differentiation of human thyroid anaplastic carcinoma cells. Oncogene 1997; 14: 729-40. 
[12] Komoike Y, Tamaki Y, Sakita I, et al. Comparative genomic hybridization defines frequent loss on $16 \mathrm{p}$ in human anaplastic thyroid carcinoma. Int J Oncol 1999; 14: 1157-62.

[13] Smallridge RC, Marlow LA, Copland JA. Anaplastic thyroid cancer: molecular pathogenesis and emerging therapies. Endocr Relat Cancer 2009; 16: 17-44.

[14] Nel CJ, van Heerden JA, Goellner JR, et al. Anaplastic carcinoma of the thyroid: A clinicopathologic study of 82 cases. Mayo Clin Proc 1985; 60: 51-8.

[15] Carcangiu ML, Steeper T, Zampi G, Rosai J. Anaplastic thyroid carcinoma: A study of 70 cases. Am J Clin Pathol 1985; 83: 135-58.

[16] Venkatesh, YSS, Ordonez, NG, Schultz, PN, et al. Anaplastic carcinoma of the thyroid: A clinicopathologic study of 121 cases. Cancer 1990; 66: 321-30.

[17] Ricarte-Filho JC, Ryder M, Chitale DA, et al. Mutational Profile of Advanced Primary and Metastatic Radioactive Iodine-Refractory Thyroid Cancers Reveals Distinct Pathogenetic Roles for BRAF, PIK3CA, and AKT1 Cancer Res 2009; 69: 4885-93.

[18] Nishiyama RH, Dunn EL, Thompson NW. Anaplastic spindle-cell and giant-cell tumors of the thyroid gland. Cancer 1972; 30: 113-27.

[19] Murakami T, Noguchi S, Murakami N, et al. Destructive thyrotoxicosis in a patient with anaplastic thyroid cancer. Endocrinol Jpn 1989; 36:905-7.

[20] Oppenheim A, Miller M, Anderson GH Jr., et al. Anaplastic thyroid cancer presenting with hyperthyroidism. Am J Med 1983; 75: 702-4.

[21] Chiacchio S, Lorenzoni A, Boni G, Rubello D, Elisei R, Mariani G. Anaplastic thyroid cancer: prevalence, diagnosis and treatment. Minerva Endocrinol 2008; 33: 341-57.
[22] Troch M, Koperek O, Scheuba C, et al. High Efficacy of Concomitant treatment of undifferentiated (anaplastic) thyroid cancer with radiation and docetaxel. J Clin Endocrinol Metab. 2010 Jun 30. [Epub ahead of print]

[23] Smallridge RC, Marlow LA, Copland JA. Anaplastic thyroid cancer: molecular pathogenesis and emerging therapies. Endocrine-Rel Cancer 2009; 16: 17-44.

[24] Miccoli P, Materazzi G, Antonelli A, Panicucci E, Frustaci G, Berti $P$. New trends in the treatment of undifferentiated carcinomas of the thyroid. Langenbecks Arch Surg 2007; 392: 397-404.

[25] Peter Lind, MD, Susanne Kohlfürst. Respective roles of thyroglobulin, radioiodine imaging, and positron emissiontomography in the assessment of thyroid cancer. Semin Nucl Med 2006; 36:194-205.

[26] Khan N, Oriuchi N, Higuchi T, Endo K. Review of fluorine-18-2fluoro-2-deoxy-D-glucose positron emission tomography (FDG-PET) in the follow-up of medullary and anaplastic thyroid carcinomas. Cancer Control 2005; 12: 254-60.

[27] Bogsrud TV, Karantanis D, Nathan MA, et al., Lowe VJ.18F-FDG PET in the management of patients with anaplastic thyroid carcinoma. Thyroid 2008; 18, 713-9.

[28] Poisson T, Deandreis D, Leboulleux S, et al. 18F-fluorodeoxyglucose positron emission tomography and computed tomography in anaplastic thyroid cancer. Eur J Nucl Med Mol Imaging 2010; 37: 2277-85.

[29] Weiping W, Steven ML, Melissa F, et al. Scanning in patients with thyroid cancer prognostic value of [18F]fluorodeoxyglucose positron emission tomographic. J Clin Endocrinol Metab 2000; 85: 1107-13.

(C) Vorster and Sathekge; Licensee Bentham Open.

This is an open access article licensed under the terms of the Creative Commons Attribution Non-Commercial License (http://creativecommons.org/licenses/by-nc/ 3.0/) which permits unrestricted, non-commercial use, distribution and reproduction in any medium, provided the work is properly cited. 